\title{
Exposure Zones Near Parasitic Elements in High Powered Antennas
}

\author{
David V. Thiel ${ }^{(1)}$ \\ (1) Centre for Wireless Monitoring and Applications, Griffith University, Nathan \\ Qld Australia. \\ E-mail: d.thiel@griffith.edu.au
}

\section{Introduction}

It is well known that a parasitic monopole located close to a radiating monopole changes the radiation properties of the antenna [1-3]. When the parasitic element acts as a reflector, the principal radiation direction is located in the radial plane through the two elements and in the opposite direction to the parasitic element. The effect is significant only when the parasitic element carries significant induced current.

It is tempting to suggest that conducting objects located behind the parasitic element will have significantly less current relative to that induced in the parasitic element, however, matrix impedance theory [2,3] for multi-element arrays assumes each element of the array is linked to every other element of the array.

In high powered transmitting antennas which use parasitic elements or have isolated conductive towers and guy lines as part of the structure, it is important to know if the region immediately behind the parasitic structures has significantly reduced radiation exposure. The reason of this investigation was to assess radiation levels with and without a human present in this zone and the effect on the radiation characteristics of the antenna. While most applications relate to VLF, LF and HF antennas where the size of a human is close to or very much less than one free space wavelength, there are also implications for UHF antennas where objects can be positioned close to the reflector elements. One example is the exposure level of an installation technician working immediately behind a Yagi-Uda antenna. The field strength immediately behind Yagi-Uda antennas was investigated previously by Ebersbach et al [4] who assumed free space in this region. Experimental field strength measurements are made remotely as humans affect the measurements.

\section{Theory}

The effect of a parasitic wire element located in the near field of a driven monopole is well established [2]. As the separation distance is decreased, the induced current increases. The induced current is approximately 180 degree out of phase with the current in the driven element. In the limit when the separation distance is zero, the driven element is effectively short circuited and there is almost no radiated field. 
Array matrix theory can be used to deduce the current in each element and so the far field radiation pattern is determined [3]. Very close to the wires, the electric and magnetic fields decrease as the cube of the radial distance and so the field strength calculations are slightly more difficult. For each straight wire antenna element, there are two electric field components $E_{\theta}$ and $E_{r}$, and one magnetic field component $H_{\varphi}$. Each field component must be summed vectorially taking into account the phase delays between the current courses. In this investigation, NEC [5] was used to determine the field strength values close to the parasitic element.

\section{Antenna Structure and Results}

Consider the simple two element monopole array shown in Fig 1. The driven element is located at $(0,0,0)$ on the $x y$ ground plane and the parasitic element is grounded and located at $\left(0, \lambda_{0} / 4,0\right)$. Both monopoles are $\lambda_{0} / 4$ in length.

The relative electric field on the ground plane in the vicinity of the elements has only a vertical component and the variation is given in Fig 2. The magnetic field is horizontal. There is a significant decrease in the electric field strength in the region immediately surrounding the parasitic element, however the magnetic field is significantly increased.

Fig 3 shows the same field distribution when an additional grounded conductive element is located in the shadow region close to the parasitic element. The element length was shorter $\left(\lambda_{0} / 10\right)$ to represent a human in this zone. There is still significant current in the human which is higher than that predicted by the electric field strength without the human present. Clearly the human form has perturbed the field in such a way that the free space field strength does not accurately predict the exposure immediately behind a parasitic.

\section{Conclusions}

This simple investigation, and results from many other experimental measurements and theoretical investigations demonstrate the following:

a) There is a region close to the parasitic element where the electric field strength is greatly reduced and the magnetic field is greatly increased.

b) The spatial rate of change of the field strength is very high. Small changes in distance can result in very large changes in electric field strength. This effect is observed in many transmitter situations.

c) The presence of an additional conductor in this zone will influence the near fields and so can influence the overall effectiveness of the antenna.

These conclusions are important because:

a) Grounded towers used in VLF, LF and HF antennas show small regions where the measured and modeled quasi-static fields are greatly reduced. Such 
regions might be defined as "safe" when radiation exposure standards [6] are applied.

b) The near field zone immediately behind Yagi-Uda antennas is not as "safe" as that predicted from far field to near field transform approximations (Ebersbach, Thiel and Leckenby [4]).

c) Antenna isolation from nearby conductors using parasitic elements placed close to the driven elements is not particularly effective when compared to a large reflecting screen.

\section{References}

[1] D.V. Thiel, S.G. O'Keefe and J.W. Lu, "Electronic beam steering in wire and patch systems using switched parasitic elements," IEEE APS International Symposium, Seattle, pp. 534-537, 1996.

[2] C. A. Balanis, Antenna Theory - Analysis and Design, 2nd ed. New York, NY: John Wiley \& Sons, 1997.

[3] D.V. Thiel and S. Smith, Switched parasitic antennas for cellular communications, Artech House, 2002.

[4] H. Ebersbach, D. V. Thiel, and M. Leckenby, "Estimation of exposure zone boundary distances from far-field antenna characteristics for finite radius wire antenna arrays," in Proc. IEEE APS International Symposium, Monterey, CA, Jun. 20-26, 2004.

[5] NecWinPro, v 1.6, Nittany Scientific, 2004.

[6] IEEE Standard for Safety Levels with Respect to Human Exposure to Radio Frequency Electromagnetic Fields, $3 \mathrm{kHz}$ to $300 \mathrm{GHz}$, Institute of Electrical and Electronics Engineers (IEEE) Std. C95.1, 2005.

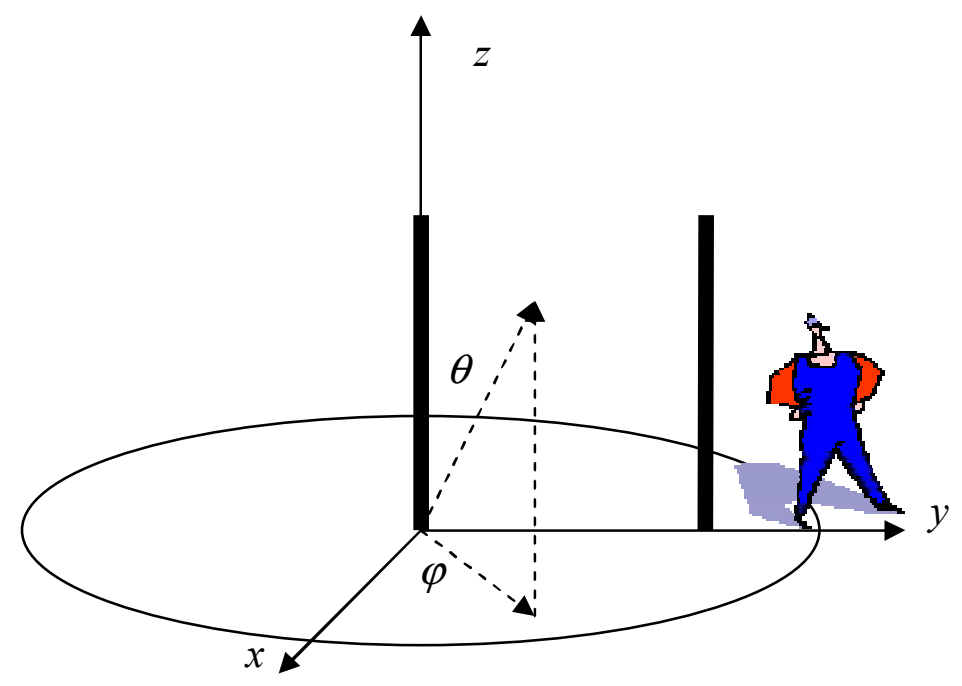

Fig 1. Monopole antenna on an infinite ground plane with a single parasitic element and a human located close to the parasitic element 


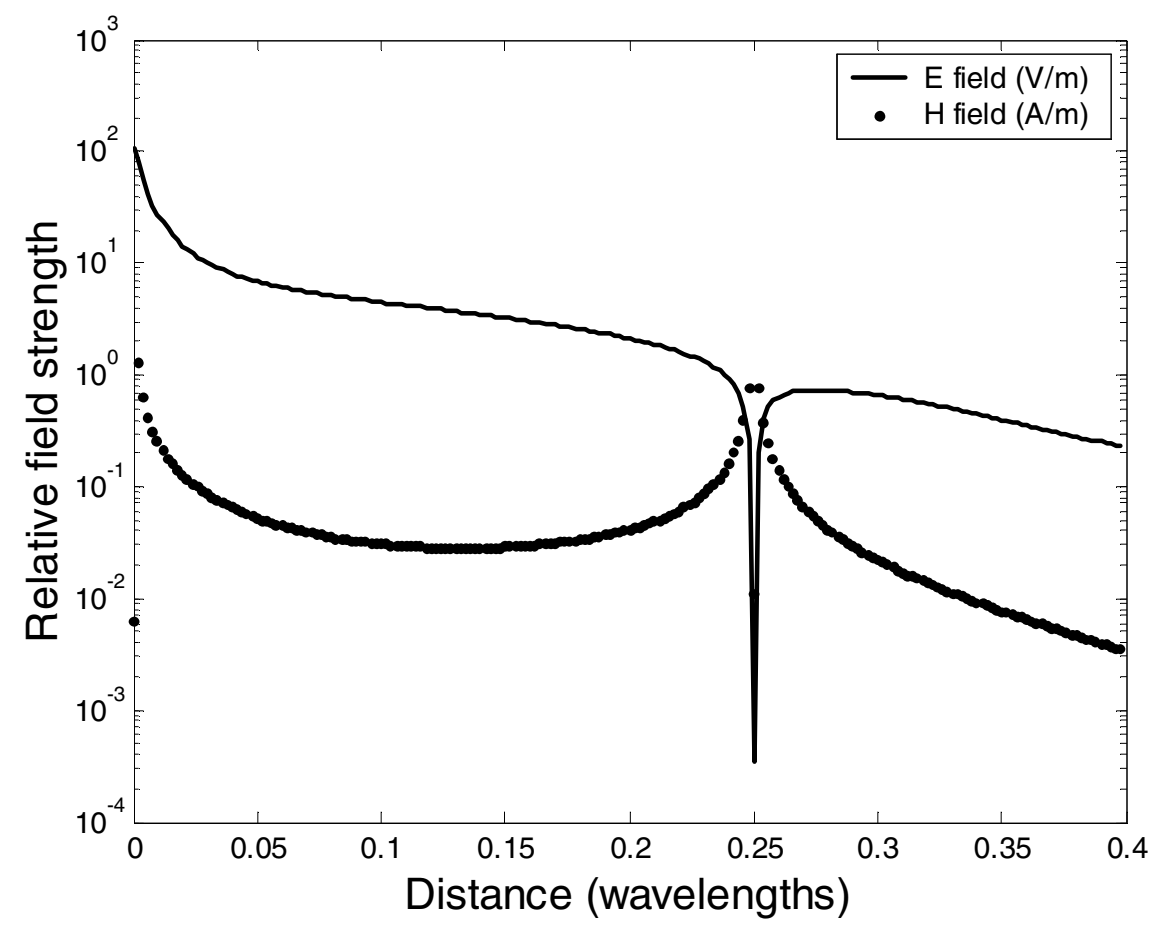

Fig 2. Relative Electric and Magnetic field strength on the $y$ axis for the antenna in Fig 1 without the human. The parasitic element is located $0.25 \lambda$.

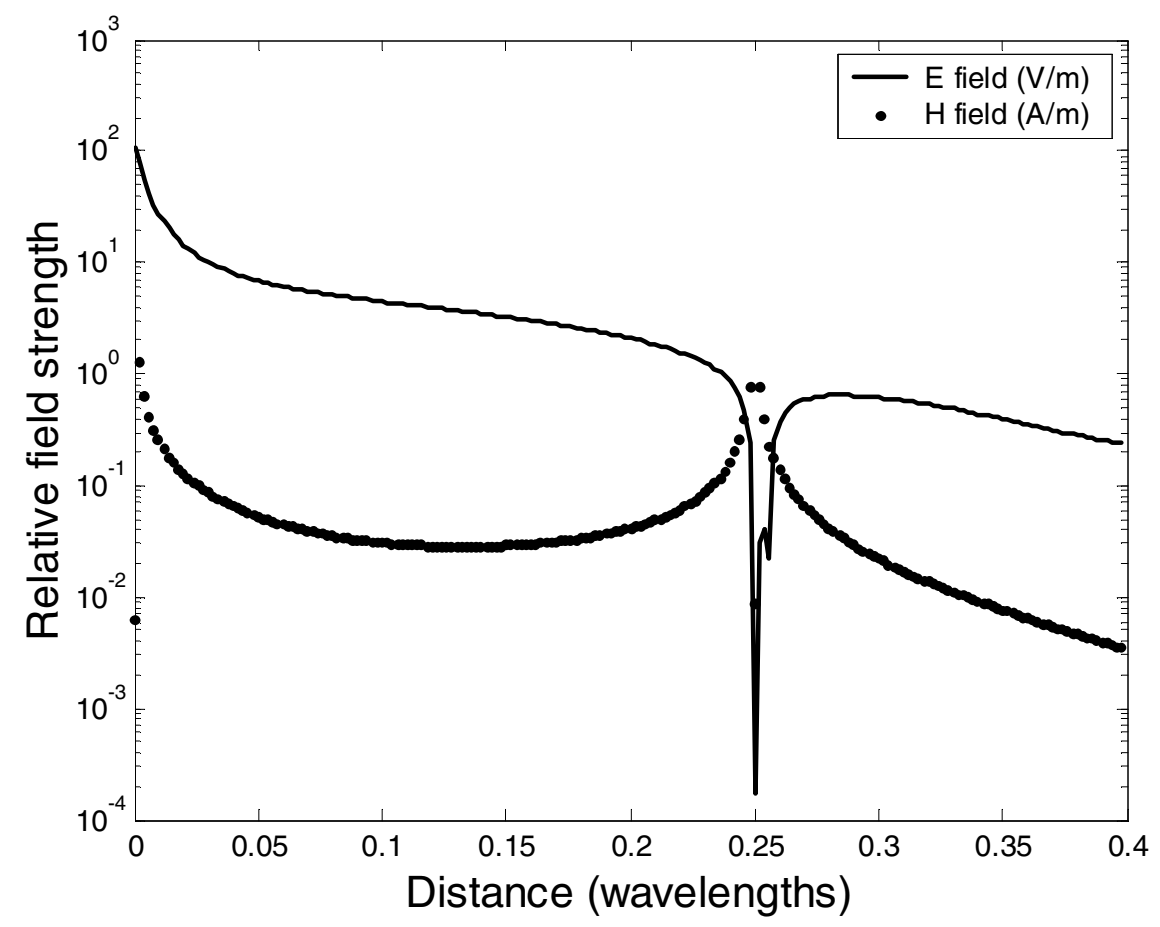

Fig 3. Relative Electric field strength contours for the antenna in Fig 1 including the human located at $0.255 \lambda_{0}$ 